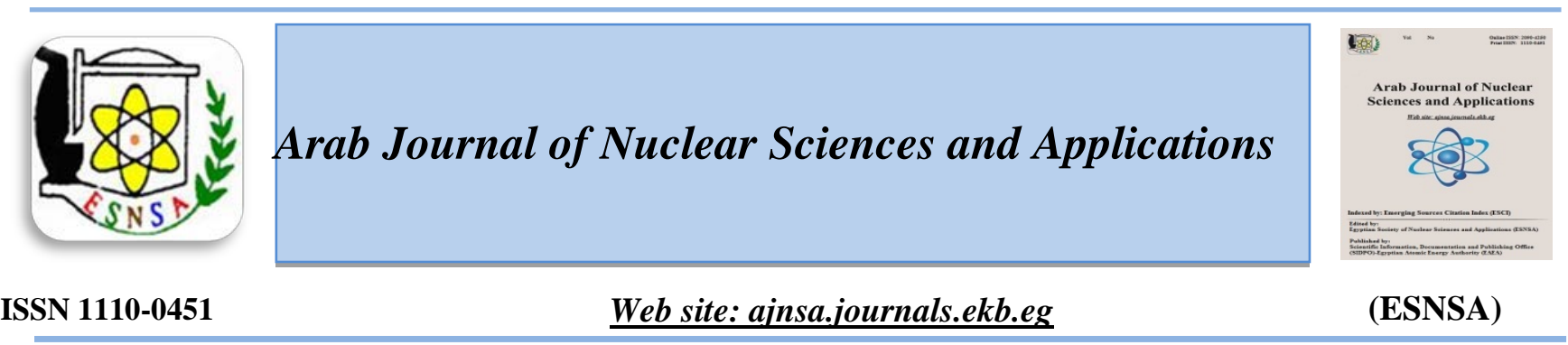

\title{
Improvement of 6 Mercaptopurine Efficiency by Encapsulated in Chitosan Nanoparticles
}

\author{
Amna Hussein ${ }^{(1)}$, Badr Y.A ${ }^{(1)}$, Samia A. Shouman ${ }^{(2)}$ and Mahmoud A. Sliem ${ }^{(1)}$ \\ ${ }^{1}$ Laser Science and Interaction, National Institute of Laser Enhanced Sciences, Cairo University \\ ${ }^{2}$ Cancer Biology Department, National Cancer Institute, Cairo University
}

\begin{abstract}
Received $13^{\text {th }}$ Jan. 20186 mercaptopurine is a cytotoxic and immune-suppressant drug. The use of this drug is limited due to its Accepted $21^{\text {st }}$ Feb. 2018 poor bioavailability and short plasma half-life. The main objective of this study is to formulate 6 mercaptopurine (6 MP) encapsulated chitosan nanoparticles (6-MP-CNPs) using Tripolyphosphate (TPP) as cross-linker for anti-cancer therapy in order to enhance bioavailability. The prepared nanoparticles were characterized using transmission electron microscopy, zeta potential. The average particle size determined through TEM was found to be $90 \pm 10 \mathrm{~nm}$ and after encapsulation the particle size had shown an obvious increase, Zeta potential 26.2 $\pm 6.35 \mathrm{mV}$. 6 MP-CNPs showed enhancement in cellular inhibition of breast cancer cell line MCF 7 compared to free 6 MP.
\end{abstract}

Keywords: Chitosan Nanoparticles/ 6 Mrcaptopurine / Cytotoxicity

\section{Introduction}

6 mercaptopurine is an effective immune suppressants and anti-cancer agents and is increasingly prescribed to treat inflammatory diseases[1] . 6-mercaptopurine was approved as an antitumor drug by Food and Drug Administration (FDA) in 1953[2] and has been widely used in the treatment of Acute Lymphoblastic Leukemia and Acute Myelocytic Leukemia [3] and other diseases such as rheumatologic disorders, prevention of rejection following organ transplantation, systemic lupus erythematosus, non-Hodgkin-lymphoma and inflammatory diseases[4]. $6 \mathrm{MP}$ is first metabolized to an active form methylated thioinosinic acid (MeTIMP) by hypoxanthine phosphoribosyl transferase (HPRT) within cell to inhibit de novo purine synthesis [5] and later converted to thioguanine, which is a DNA intercalating agent. Incorporation of thioguanine induces cytotoxicity mediated via G2/M and/or S phase arrest[6]. However; $6 \mathrm{MP}$ undergoes a complex biotransformation rendering the drug inactive. Therefore, $6 \mathrm{MP}$ undergoes extensive first pass catabolism by XO (Xanthine oxidase) and TPMT (thiopurine $\mathrm{S}$ methyl transferase) limiting their bioavailability. So this could lead to a lower bioavailability (about 16\%)[7] . Nanocarriers like liposomes, dendrimers, nanofibers, nanotubes and nanoparticles are essentially required for providing and maintaining desired concentration with minimal toxicity[8]. In order to justify these issues, nanotechnology has been mainly dealing with the synthesis of nanomaterials of variable size, shape, surface charge and narrow size distribution, which have been typically explored, as they represent excellent carriers for the integration of hydrophobic 6 MP [9]. There are several limitations of these carrier systems, such as expensive or conservative synthesis procedure, poor ability to control the size distribution, instability in biological fluids, inadequate tissue distribution, low drug loading efficiency, lack of bioavailability and biodegradation with precursor

Corresponding author: amna hussein090@yahoo.com

DOI: 10.21608/ajnsa.2018.2630.1040

(C) Scientific Information, Documentation and Publishing Office (SIDPO)-EAEA 
material toxicity [10]. In order to address these disadvantages and improving the oral bioavailability, chitosan was selected as a natural, biodegradable, biocompatible, non-toxic, nonimmunogenic and inexpensive mucoadhesive biopolymer [11]. Chitosan is a mucoadhesive polymer having an affinity to bind with intestinal mucosa and leading to improving the residence time of drugs in the intestinal lumen and, consequently, enhance their bioavailability. Recently, Chitosan nanoparticles were found to be promising carriers for controlled-release drug delivery systems [12]. Among a variety of methods developed to prepare chitosan nanoparticles, ionic gelation technique have attracted considerable attention due to its non-toxic, organic solvent free, convenient and controllable process [13]. Ionic gelation technique is based on the ionic interactions between the positively charged primary amino groups of chitosan and the negatively charged groups of polyanion, such as sodium tripolyphosphate (TPP). This physical cross-linking process, not only avoids the use of chemical cross-linking agents and emulsifying agents which are often toxic to organisms, but also prevents the possibility of damage to drugs, particularly biological agents [14].

\section{Materials and Method}

Chemicals are obtained from Sigma Aldrich Chemical Co., St. Louis, Mo, U.S.A. These materials include 6 mecaptopurine, low molecular weight chitosan and sodium tri poly phosp .Human breast carcinoma cell line (MCF 7) was obtained from the American Type Culture Collection (ATCC, MO, USA). The tumor cell line was propagated and maintained by serial subculturing in RPMI-164a0 medium containing $10 \%$ FBS and 1\% penicillin/ streptomycin.

\section{Preparation of chitosan nanoparticles:}

Chitosan nanoparticles were produced based on ionic gelation method of TPP with chitosan [15]: Chitosan was dissolved at $0.6 \%(\mathrm{w} / \mathrm{v})$ with $1 \%$ $(\mathrm{v} / \mathrm{v})$ acetic acid, the solution was raised to $\mathrm{pH}$ 4.6-4.8 with $1 \mathrm{~N} \mathrm{NaOH}$. Chitosan nanoparticles formed spontaneously upon the addition of an aqueous tripolyphosphate solution $(0.4 \%, \mathrm{w} / \mathrm{v})$. The prepared chitosan solutions were flushedmixed with TPP solutions under magnetic stirring at $800 \mathrm{rpm}$ for $30 \mathrm{~min}$ at room temperature.
Chitosan was mixed with TPP in a volumetric ratio of $3: 1$.

\section{Preparation of 6 MP encapsulated chitosan nanoparticles}

Chitosan was dissolved at $0.6 \%(\mathrm{w} / \mathrm{v})$ with $1 \%$ $(\mathrm{v} / \mathrm{v})$ acetic acid solution. $5 \mathrm{ml}$ of the Prepared chitosan solutions were mixed with $1 \mathrm{ml}$ of $10^{-4} \mathrm{M}$ 6MPsolutions (6 MP dissolved in water). The $\mathrm{PH}$ was raised to $4.6-4.8$ with $1 \mathrm{~N} \mathrm{NaOH}$. The Prepared 6MP containing chitosan solutions were flushed mixed with $0.4 \%(\mathrm{w} / \mathrm{v})$ TPP solutions in a volume ratio of $(3: 1)(\mathrm{v} / \mathrm{v})($ chitosan : TPP). The nanoparticle suspension was gently stirred at 800 rpm for $30 \mathrm{~min}$ at room temperature .

In vitro study for the potential cytotoxicity of $6 \mathrm{MP}$ and 6 MP encapsulated Chitosan nanoparticles on MCF 7 breast cancer cell line using sulphorhodamine-B (SRB) assay

This method was carried out according to that of Skehan et al. (1990) [16]. Cells were seeded in 96well microtiter plates at a concentration of $5 \times 10^{3}$ Cells/well in a fresh medium and left to attach to the plates for 24 hrs. Cells were incubated with the same concentrations of free $6 \mathrm{MP}$ and $6 \mathrm{MP}$ encapsulated Chitosan NPs (1.5 x 10 $0^{-6}, 3.1 \times 10^{-6}$, $6.2 \times 10^{-6}, 12.5 \times 10^{-6} \mathrm{M}$ ) for $48 \mathrm{hrs}$. The cells were fixed with cold $50 \%$ trichloroacetic acid for $1 \mathrm{hr}$ at $4^{0} \mathrm{C}$ and washed with distilled water and stained with $0.4 \%$ SRB. The wells were washed with $1 \%$ acetic acid, air-dried and the dye was solubilized with $10 \mathrm{mM}$ tris base (pH 10.5) using shaker. The optical density (O.D.) of each well was measured spectrophotometrically at $564 \mathrm{~nm}$ with an ELIZA microplate reader (Meter tech. $\Sigma 960$, U.S.A.). The mean background absorbance was automatically subtracted and the mean values of each drug concentration were calculated. The percentage of cell survival was calculated as follows: Survival fraction = O.D (treated cells)/ O.D (control cells). The $\mathrm{IC}_{50}$ values (the concentrations that produced $50 \%$ inhibition of cell growth) was measured. The experiment was repeated 3 times independently and each concentration was repeated three times.

\section{Statistical analysis}

Data are expressed as mean \pm SD. Statistical analysis was carried out using Graph Pad Software Prism version 5. The statistical analysis of the transfection assays data was done using Tukey 
multiple comparison test. Differences were considered statistically significant when $\mathrm{p} \leq 0.01$.

\section{Results}

The characterization of the prepared chitosan nanoparticles:

The mean size distribution and zeta potential of the prepared chitosan nanoparticle suspension were analyzed using the Zetasizer analysis Table (1). The mean particle size was approximately $90 \pm 10$ $\mathrm{nm}$. These nanoparticles had a relatively narrow particle size distribution with PDI value 0.4. Zeta potential, this is the surface charge that can greatly influence particle stability in suspension through the electrostatic repulsion between particles. It can also determine nanoparticle interaction in vitro with the cell membrane of cancer cell which is usually negatively charged. Table (1) shows that the surface of chitosan nanoparticles has a positive charge $26 \mathrm{mV}$. The chitosan nanoparticles showed a spherical morphology and uniform distribution with diameters in the range of $80-110 \mathrm{~nm}$. The TEM images of the 6 MP encapsulated chitosan nanoparticles showed slightly altered morphology (Figure 3) with an increase in diameter ranging from 110 - $225 \mathrm{~nm}$ which may be due to the interaction and encapsulation of 6 MP with Chitosan -TPP ionic gelation.

Table (1) the average size and zeta potential of 3:1 chitosan to TPP ratio

\begin{tabular}{|c|c|c|c|}
\hline $\begin{array}{c}\text { Chitosan: } \\
\text { TPP ratio } \\
(\mathrm{v} / \mathrm{v})\end{array}$ & $\begin{array}{c}\text { Average } \\
\text { particle } \\
\text { Size } \\
(\mathrm{nm})\end{array}$ & $\begin{array}{c}\text { Polydispersity } \\
\text { index (PDI) }\end{array}$ & $\begin{array}{c}\text { Zeta } \\
\text { potential } \\
(\mathrm{mV}) \\
(\text { Mean } \pm \\
\text { SD) }\end{array}$ \\
\hline $3: 1$ & $90 \pm 10$ & 0.4 & $26.2 \pm 6.35$ \\
\hline
\end{tabular}

In vitro cytotoxicity of 6 MP encapsulated chitosan NPs on MCF 7:

Figure (4) reveals the effect of different concentrations of $6 \mathrm{MP}$ and $6 \mathrm{MP}$ encapsulated chitosan NPs $(1.5,3.1,6.2$ and $12.5 \mu \mathrm{m})$ on the survival percentage of breast carcinoma cell line (MCF 7) after 48 hr. exposure to the drug. There was a concentration dependent decrease in cellular proliferation compared to its respective control. It is shown that after $48 \mathrm{hr}$. $6 \mathrm{MP}$ produced a decrease in cell survival by $8 \%$ at $3.1 \mu \mathrm{m}$ compared to its control, while 6 MP encapsulated chitosan NPs produced a significant decrease in cell survival compared to its control reaching a maximum cytotoxicity 55\% at $6.2 \mu \mathrm{m}$. Figure (4) shows that the $\mathrm{IC}_{50}$ for $6 \mathrm{MP}$ encapsulated Chitosan NPs value of was $5.5 \mu \mathrm{m}$.

\section{Discussion}

Chitosan nanoparticles have an important role for drug delivery concerning cancer treatments. The preparation of chitosan NPs is based on an ionic gelation interaction between positively charged chitosan and negatively charged tripolyphosphate (TPP). Formation of nanoparticles occurs spontaneously through the formation of intra- and intermolecular cross-linking under a constant stirring at ambient temperature ${ }^{[17]}$. TPP are a multivalent anion that possesses negative charges; chitosan in acidic solution has amino groups that can undergo protonation. During the preparation process, TPP is electrostatically attracted to the $\mathrm{NH} 3^{+}$groups in chitosan to produce ionically crosslinked chitosan NPs ${ }^{[18]}$. TPP has often been used to prepare chitosan nanoparticles because TPP is nontoxic, multivalent and able to form gels through ionic interactions. The interaction can be controlled by the charge density of TPP and chitosan, which is dependent on the $\mathrm{pH}$ of the solution ${ }^{[19]}$. Important properties of nanoparticles such as particle size or surface charge can be easily manipulated by changing parameters such as concentration of chitosan, chitosan-to-polyanion concentration ratio, and solution $\mathrm{pH}^{[20]}$.

The cytotoxicity of 6 MP encapsulated chitosan NPs may be improved due to better accumulation of drug at its site of action due to targeted delivery. A possible explanation for the activity enhancement of 6 MP encapsulated chitosan NPs may be due to internalization of 6 MP encapsulated chitosan NPs by an endocytosis mechanism. Generally, nanoparticles are nonspecifically internalized into cells via endocytosis or phagocytosis compared to the passive diffusion mechanism of free drugs into cells ${ }^{[21]}$. This efficient cytotoxic effect could be due to the nanoparticles unique properties such as small size, altered shape, high surface area-tovolume ratio and thus, many of the physical properties of the nanoparticles such as solubility and stability are dominated by the nature of the nanoparticle surface functionalization and are critical for their intended biological functions ${ }^{[22]}$.

\section{Conclusions}

With increasing necessity to develop drugs with greater efficiency to target cancer cells specifically 
and improve chemotherapeutic efficiency, there is a constant requirement to develop or improve drug delivery strategies. Therefore, the present study aimed to develop nanoparticle synthesis procedure that facilitates drug loading and preferentially makes cancer cells more responsive. In this study, 6 MP modified chitosan nanoparticles were prepared using ion exchange method for drug delivery increasing chemotherapeutic effect. Based on the results obtained from the study. It can be concluded that the developed novel nanoparticles will serve as valuable anticancer drug delivery system.
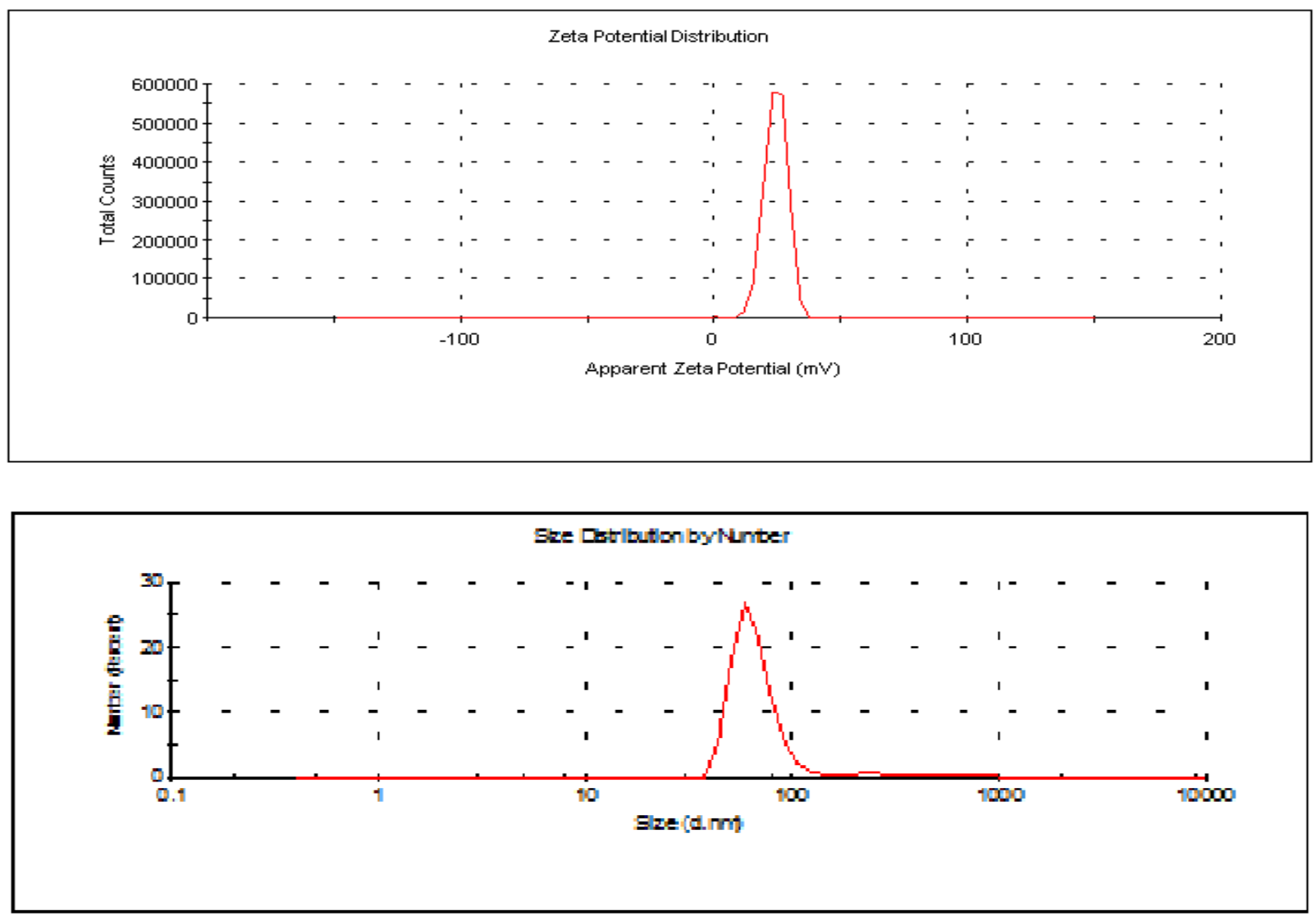

Figure (1): Zeta sizer and potential of chitosan nanoparticles chitosan: TPP was 3:1

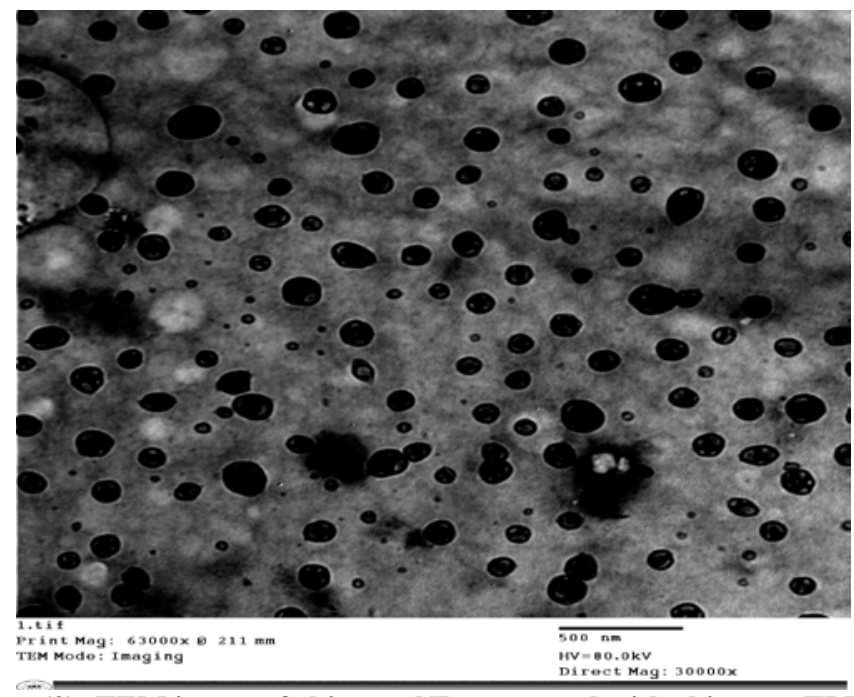

Figure (2): TEM image of chitosan NPs prepared with chitosan: TPP 3:1 


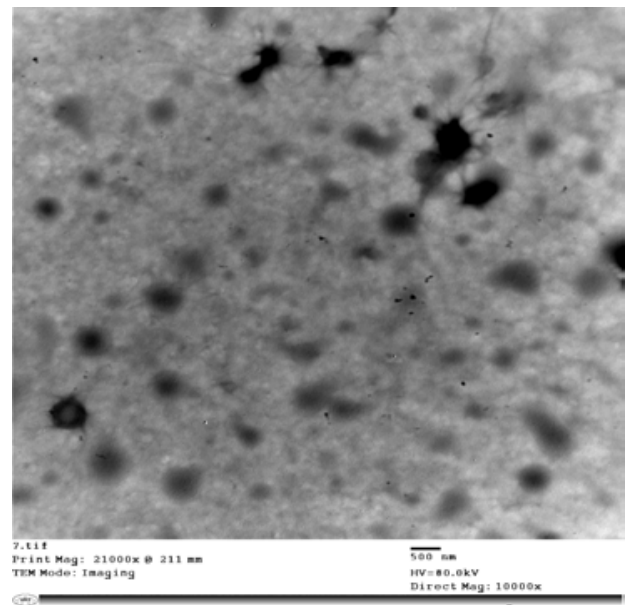

Figure (3): TEM image of 6 MP encapsulated chitosan NPs prepared with chitosan: TPP 3:1
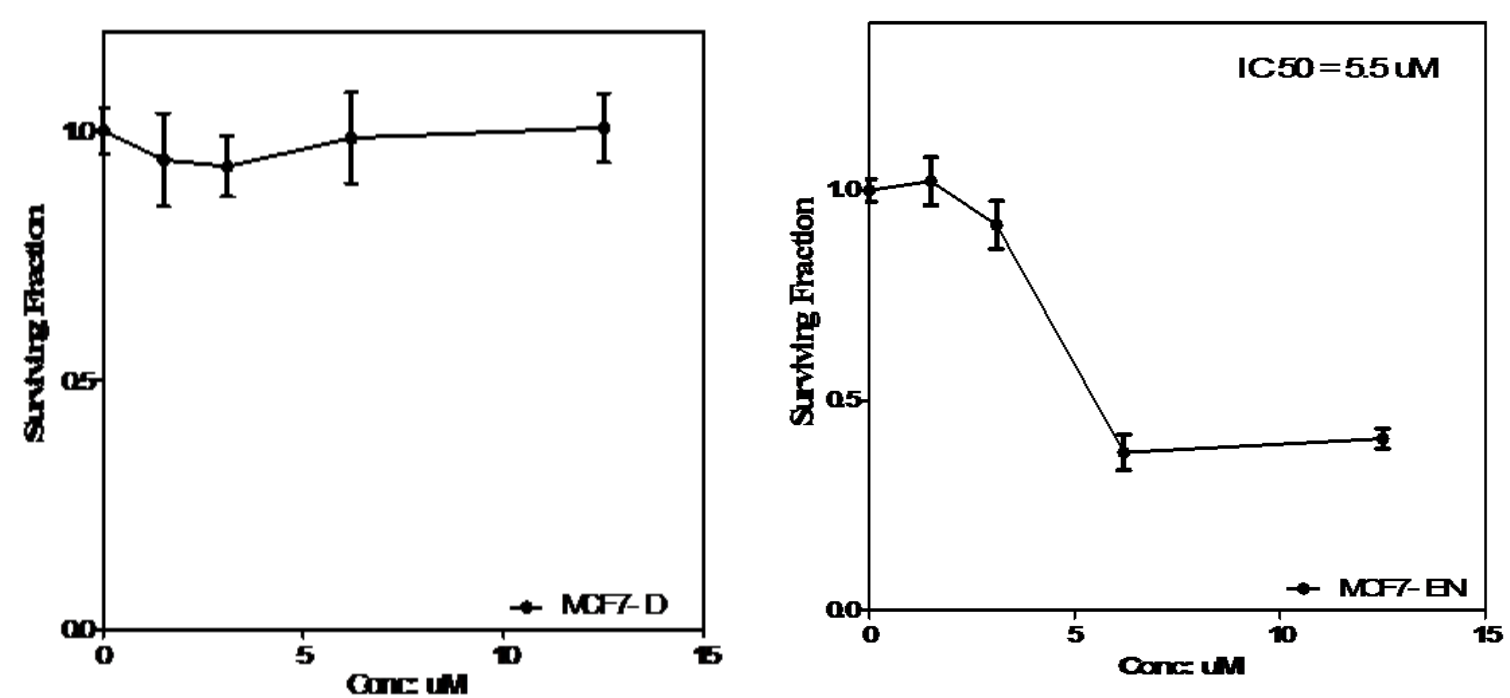

Figure (4): Cytotoxicity of different concentration of 6 MP and 6 MP encapsulated chitosan NPs on MCF 7 cell line after 48 hr

\section{References}

1- EHTESHAMI, M., RASOULZADEH, F., MAHBOOB, S. and RASHIDI, M.R. Characterization of 6-mercaptopurine binding to bovine serum albumin and its displacement from the binding sites by quercetin and rutin. J. Lumin., 135:164-169, (2013).

2- CUIN, A., MASSABNI, A.C., PEREIRA, G.A., LEITE, C.Q.F., PAVAN, F.R., COSTA, R.S., HEINRICH, T.A. and COSTA-NETO, C.M. 6mercaptopurine complexes with silver and gold ions: Anti-tuberculosis and anti-cancer activities. Biomed. Pharmacother., 65:334-338, (2011).

3-SUN, H., WANG, T., LIU, X. and CHEN, P. A sensitive inhibition chemiluminescence method for the determination of 6-mercaptopurine in tablet and biological fluid using the reaction of luminol-Ag
(III) complex in alkaline medium. J. Lumin., 134:154-159, (2013).

4- KEVADIYA, B.D., CHETTIAR, S.S., RAJKUMAR, S., BAJAJ, H.C., GOSAI, K.A. and BRAHMBHATT, H. Evaluation of clay/poly (llactide) micro composites as anticancer drug, 6mercaptopurine reservoir through in vitro cytotoxicity, oxidative stress markers and in vivo pharmacokinetics. Colloids Surf. B, 112:400-407, (2013).

5- PANETTA, J.C., EVANS, W.E. and CHEOK, H. Mechanistic mathematical modelling of mercaptopurine effects on cell cycle of human acute lymphoblastic leukaemia cells. Br. J. Cancer, 94:93100, (2006).

6- MILEK, M., KUZELICKI, N.K., SMID, A. and MLINARIC-RASCAN, I. Sadenosylmethionine 
regulates thiopurine methyl transferase activity and decreases 6-mercaptopurine cytotoxicity in MOLT lymphoblasts. Biochem. Pharmacol., 77:1845-1853, (2009).

7-CHEOK, M.H. and EVANS, W.E. Acute lymphoblastic leukaemia: a model for the pharmacogenomics of cancer therapy. Nat. Rev. Cancer, 6:117, (2006).

8- KEVADIYA, B.D., PATEL, T.A., JHALA, D.D., THUMBAR, R.P., BRAHMBHATT, H., PANDYA, M.P., RAJKUMAR, S., JOSHI, G.V., GADHIA, P.K., TRIPATHI, C.B. and BAJAJ, H.C. Layered inorganic nanocomposites: a promising carrier for 5fluorouracil (5-FU). Eur. J. Pharm. Biopharm., 81:91, (2012).

9- WILCZEWSKA, A.Z., NIEMIROWICZ, K., MARKIEWICZ, K.H. and CAR, H. Nanoparticles as drug delivery systems. Pharmacol. Rep., 64:1020, (2012).

10-KEVADIYA, B.D., CHETTIAR, S.S., RAJKUMAR, S., BAJAJ, H.C., GOSAI, K.A. and BRAHMBHATT, H. Evaluation of clay/poly (llactide) micro composites as anticancer drug, 6 mercaptopurine reservoir through in vitro cytotoxicity, oxidative stress markers and in vivo pharmacokinetics. Colloids Surf. B, 112:400-407, (2013).

11-SONIA, T.A. and CHANDRA, P.S. Chitosan and its derivatives for Drug Delivery Perspective. Adv. Polym. Sci., 243:23-54, (2011).

12- YANG, H.C. and HON, M.H. The effect of the molecular weight of Chitosan nanoparticles and its application on drug delivery. Microchemical J., 92:87-91, (2009).

13-AGNIHOTRI, S.A., MALLIKARJUNA, N.N. and AMINABHAVI, T.M. Recent advances on chitosanbased micro and nanoparticles in drug delivery. $J$. Control Release, 100:5-28, (2004).

14-BERGER, J., REIST, M., MAYER, J.M., FELT, O., PEPPAS, N.A. and GURNY, R. Structure and interactions in covalently and ionically crosslinked Chitosan hydrogels for biomedical applications. Eur. J. Pharm. Biopharm., 57:19-34, (2004).

15- R. Seda T1־glı Aydın1, and Mehlika Pulat 5Fluorouracil Encapsulated Chitosan Nanoparticles for pH-Stimulated Drug Delivery: Evaluation of Controlled Release Kinetics Hindawi Publishing Corporation Journal of Nanomaterials Volume, Article ID 313961, 10 pages (2012).

16-Skehan P., Storeng R., Scudiero D. Monks A, McMahon VD. Warren JT, Bokesch H, Kenney S and Boyd MR. New colorimetric cytotoxicity assay for anticancer-drug screening. J. Natl. Cancer Inst.; 82: 1107, (1990).

17- Qi, L., Xu, Z., Jiang, X., Hu, C. and Zou, X. "Preparation and antibacterial activity of chitosan nanoparticles," Carbohydrate Research, 339(16) 2693-2700, (2004).

Arab J. Nucl. Sci. \& Applic. Vol. 51, No.2 (2018)
18- Yang, H.C. and Hon, M. H. "The effect of the molecular weight of chitosan nanoparticles and its application on drug delivery," Microchemical Journal, 92(1)87-91, (2009).

19-Li-Ming Zhao1, Lu-E Shi2, Zhi-Liang Zhang2, JianMin Chen2, Dong-Dong Shi2, Jie Yang2 and ZhenXing Tang PREPARATION AND APPLICATION OF CHITOSAN NANOPARTICLES AND NANOFIBERS Brazilian Journal of Chemical Engineering Vol. 28, No. 03, pp. 353 - 362, July September.

20-Q. Gan, T. Wang, C. Cochrane, and P. Mc Carron, "Modulation of surface charge, particle size and morphological properties of chitosan-TPP nanoparticles intended for gene delivery," 2005Colloids and Surfaces B, vol. 44, no. 2-3, pp. 65-73, (2011).

21- Sadaf Aghevlian • Reza Yousefi - Reza Faghihi • Abdolkarim Abbaspour • Ali Niazi • Mansooreh Jaberipour - Ahmad Hosseini. The improvement of anti-proliferation activity against breast cancer cell line of thioguanine by gold nanoparticles Med Chem Res DOI 10.1007/s00044-012-0030-12, (2012).

22- LIU, Y., TAN, J., THOMAS, A., OU-YANG, D. and MUZYKANTOV, V.R. The shape of things to come: importance of design in nanotechnology for drug delivery. Ther. Deliv., 3:181-194, (2012). 